\title{
A Facile Solvent Free Synthesis of 3-arylidenechroman-4-ones Using Grinding Technique
}

\author{
SURESH KUMAR* and JAGDISH K. MAKRANDI ${ }^{1}$ \\ * Department of Chemistry, Kurukshetra University Kurukshetra (India)-136119 \\ ${ }^{1}$ Department of Chemistry, M.D. University Rohtak (India)-124001 \\ duaskchem@gmail.com
}

Received 09 July 2011; Accepted 05 September 2011

\begin{abstract}
An efficient method for the synthesis of 3-arylidenechroman-4-ones has been developed under solvent free conditions using grinding technique. Grinding of variously substituted chroman-4-ones with aromatic aldehydes in presence of anhydrous barium hydroxide at room temperature give 3arylidenechroman-4-ones in high yield (75-92\%). Products are obtained by just acidification of the reaction mixture in ice cold water. Reaction in solid state, with enhanced rate, high selectivity and manipulative simplicity are the attractive features of this environmentally benign protocol. The chroman-4one derivatives required for the reaction have been obtained by polyphosphoric acid (PPA) catalysed cyclisation of phenoxypropanoic acids under microwave irradiations.
\end{abstract}

Keywords: 3-Arylidenechroman-4-ones, chroman-4-ones, phenoxypropanoic acids, anhydrous barium hydroxide, aromatic aldehydes, grinding.

\section{Introduction}

3-Arylidenechroman-4-ones belong to homoisoflavanoids, a class of naturally occurring oxygen heterocyclic compounds, with C-16 skelton ${ }^{1}$. Chroman-4-one derivatives are known to exhibit a broad range of pharmacological activities ${ }^{1,2}$ including anti-human-immune deficiency virus (HIV-I) properties ${ }^{2}$ that causes acquired immune deficiency syndrome (AIDS). 3-Benzylidenechroman-4-ones have been reported to inhibit the growth of the sporogenesis and the enzymes involved in the infection mechanism of phytophtora parasitica $^{3}$ and also show antifungal activity ${ }^{4}$. These have also been found to display antiinflammatory, analgesic, platelet anti aggregating ${ }^{5}$, hypocholesterolemic ${ }^{6}$ and antioxidant activities $^{7}$. These are also used for the synthesis of some selective ligands required for 
antiestrogen-binding $\operatorname{sites}^{8}$ and as precursors for the synthesis of various other homoisoflavanoids?.

The general synthesis of 3-arylidenechroman-4-ones involves the condensation of chroman-4-ones with aromatic aldehydes in the presence of acidic or basic reagents ${ }^{10}$. But the use of acidic reagents for the condensation is usually preferred ${ }^{11,12}$ as the chroman-4-one ring is more stable to acidic reagents ${ }^{13}$. Use of bases such as alcoholic $\mathrm{KOH}, \mathrm{NaOH}$, $\mathrm{NaOCH}_{3}$, piperidine and acetic anhydride ${ }^{10,14}$ have also been reported for the synthesis of these compounds. In a recent approach Amberlyst-15 under MW irradiations have been reported for this reaction ${ }^{15}$. Ring closure of an acrylic acid derivative with trifuoroacetic anhydride in dichloromethane medium is also a route for the synthesis of 3arylidenechroman-4-ones, but is not of much interest ${ }^{16}$.

Degradation of ecosystem due to toxic nature of chemicals used, poor yields, formation of isomeric products, complexity of the procedure and high temperature required for the reaction, are the major limitations with which the reported methods suffer. In these days, much emphasis is being laid by the chemists to develop synthetic procedures which could avoid the use of toxic and hazardous chemicals including solvents ${ }^{17}$. Grinding technique, due to its operational simplicity, now has got the attention of the chemists as an important tool for green synthesis ${ }^{18}$. In continuation of our work to develop eco-friendly organic transformations ${ }^{19}$, here in we are reporting, a facile solvent free green synthesis of 3arylidenechroman-4-ones using grinding technique. (Scheme 1).

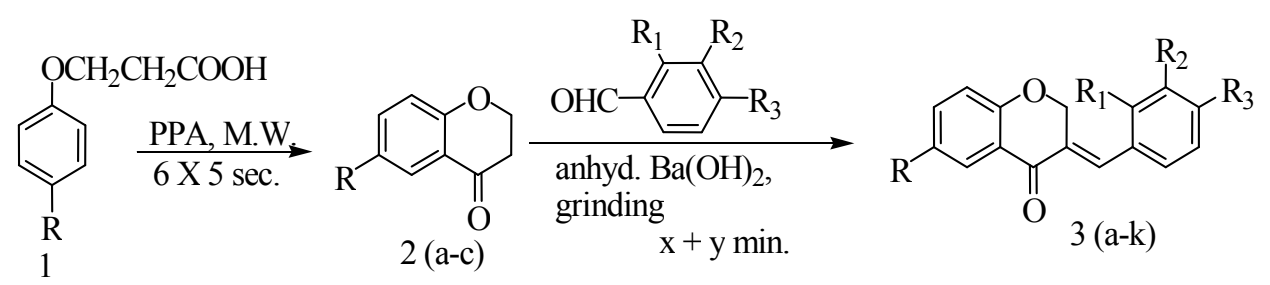

Scheme 1. Synthesis of 3-arylidenechroman-4-ones.

\section{Experimental}

Grinding in all the reaction was carried out in porcelain mortar with pestle. Melting points were determined in open capillary tubes and are uncorrected. The compounds were characterized from their spectral data (IR \& ${ }^{1} \mathrm{H}-\mathrm{NMR}$ ) and by comparison with authentic sample. The ${ }^{1} \mathrm{H}$ NMR spectra were recorded on Bruker Avance II 400 spectrometer at 400 $\mathrm{MHz}$ in $\mathrm{CDCl}_{3}$ using TMS as internal standard. Chemical shifts $(\delta)$ are reported in ppm and coupling constant in Hz. The IR spectra were recorded using Perkin Elmer spectrometer ( $\mathrm{KBr}$ plates). Reaction involving the use of microwaves were carried out in a domestic microwave oven (Samsung output energy $900 \mathrm{~W}$, frequency $2450 \mathrm{MHz}$, with temperature control arrangement No. CE $118 \mathrm{KF}$ ) using $40 \%$ power for all the experiments. 


\section{General procedure}

Synthesis of chroman-4-ones (2 a-c)

Phenoxypropanoic acid (15 g) was added to freshly prepared polyphosphoric acid (10 ml) in a round bottom flask fitted with guard tube and was thoroughly mixed by glass rod. The resulting mixture was then irradiated with microwaves for 30 seconds $(6 \times 5 \mathrm{sec}$.) using 40 $\%$ power of the oven, with shaking after each interval of 5 seconds. Pasty residue so obtained was decomposed with crushed ice $(50 \mathrm{~g})$. Cold suspension was then extracted with diethyl ether, washed with $10 \%$ aq. sodium bicarbonate $(2 \times 25 \mathrm{ml})$ followed by water and dried over magnesium sulphate. The solvent was removed by distillation and residue thus obtained was recrystallised from petroleum ether-diethyl ether mixture to give product i.e. chroman-4-one derivatives (2 a-c).

\section{Synthesis of 3-arylidenechroman-4-ones (3a-k)}

A mixture of chroman-4-one $(4 \mathrm{mmol})$, aromatic aldehyde $(4.1 \mathrm{mmol})$ and anhydrous barium hydroxide $(2.5 \mathrm{~g})$ was taken in a mortar and ground well with pestle for 5 minutes. Progress of the reaction was checked on TLC. For completion of the reaction, reaction mixture was left at room temperature for 5 minutes. It was then worked up by acidifying the reaction mixture in ice cold conditions, when solid residue was separated out which was suction filtered, washed with water and recrystallised from petroleum etherdiethyl ether mixture to give 3-arylidenechroman-4-one (3 a-k). Spectral data of the compounds synthesized are given below.

6-methyl-3-(4'-methylbenzylidene) chroman-4-one (3e):

IR (KBr): $1667 \mathrm{~cm}^{-1}(\mathrm{C}=\mathrm{O})$.

${ }^{1} \mathrm{H}-\mathrm{NMR}\left(\mathrm{CDCl}_{3}\right): \delta 3.04 \& 3.30\left(\right.$ each s, $\left.6 \mathrm{H}, 2 \times \mathrm{CH}_{3}\right), 5.33\left(\mathrm{~d}, 2 \mathrm{H},-\mathrm{OCH}_{2^{-}}, \mathrm{J}=1.84 \mathrm{~Hz}\right.$ ), $6.86(\mathrm{~d}, \mathrm{~J}=8.4 \mathrm{~Hz}, 1 \mathrm{H}, \mathrm{H}-8), 7.20-7.31$ (m, 5H, H-7, H-2', H-3', H-5', H-6'), 7.81 (d, J = $1.80 \mathrm{~Hz}, 1 \mathrm{H}, \mathrm{H}-5), 7.55(\mathrm{~s}, 1 \mathrm{H},=\mathrm{CH}-\mathrm{Ph})$.

6-methyl-3-(4'-methoxybenzylidene)chroman-4-one (3f):

IR (KBr): $1667 \mathrm{~cm}^{-1}(\mathrm{C}=\mathrm{O})$.

${ }^{1} \mathrm{H}-\mathrm{NMR}\left(\mathrm{CDCl}_{3}\right): \delta 2.34\left(\mathrm{~s}, 3 \mathrm{H}, \mathrm{CH}_{3}\right), 3.84\left(\mathrm{~s}, 3 \mathrm{H}, \mathrm{OCH}_{3}\right), 5.35\left(\mathrm{~d}, \mathrm{~J}=1.8 \mathrm{~Hz}, 2 \mathrm{H},-\mathrm{O}-\mathrm{CH}_{2}{ }^{-}\right.$ ), 6.87 (d, J = 8.4 Hz, 1H, H-8), $6.8-7.59$ (m, 5H, H-7, H-2' $\left., \mathrm{H}^{-} 3^{\prime}, \mathrm{H}_{-} 5^{\prime}, \mathrm{H}-6^{\prime}\right), 7.74$ (d, 1H, $\mathrm{H}-5, \mathrm{~J}=1.8 \mathrm{~Hz}), 7.85(\mathrm{~s}, 1 \mathrm{H}=\mathrm{CH}-)$.

\section{6-bromo-3-benzylidenechroman-4-one (3g):}

IR (KBr): $1670 \mathrm{~cm}^{-1}(\mathrm{C}=\mathrm{O})$.

${ }^{1} \mathrm{H}-\mathrm{NMR}\left(\mathrm{CDCl}_{3}\right): \delta 5.35\left(\mathrm{~d}, \mathrm{~J}=1.84 \mathrm{~Hz}, 2 \mathrm{H},-\mathrm{OCH}_{2}-\right), 6.88(\mathrm{~d}, \mathrm{~J}=8.8 \mathrm{~Hz}, 1 \mathrm{H}, \mathrm{H}-8), 7.26-$

7.32 (m, 3H, H-3', H-4', H-5'), 7.44-7.46 (m, 2H, H-2', H-6' ), 7.55-7.58 (dd, J = 2.52 Hz, \&

$8.76 \mathrm{~Hz}, 1 \mathrm{H}, \mathrm{H}-7), 7.89$ (s, 1H, = CH-), 8.13 (s, 1H, H-5).

6-bromo-3-(4'-methoxybenzylidene) chroman-4-one (3h):

IR (KBr): $1670 \mathrm{~cm}^{-1}(\mathrm{C}=\mathrm{O})$.

${ }^{1} \mathrm{H}-\mathrm{NMR}\left(\mathrm{CDCl}_{3}\right): \delta 3.86\left(\mathrm{~s}, 3 \mathrm{H}, \mathrm{OCH}_{3}\right), 5.37\left(\mathrm{~d}, \mathrm{~J}=1.8 \mathrm{~Hz}, 2 \mathrm{H},-\mathrm{OCH}_{2}-\right), 6.87(\mathrm{~d}, \mathrm{~J}=8.8$ $\mathrm{Hz}, 1 \mathrm{H}, \mathrm{H}-8), 6.96-6.98$ (m, 2H, H-3', H-5'), 7.26-7.29 (m, 2H, H-2', H-6'), 7.53 (dd, J = $2.56 \& 8.8 \mathrm{~Hz}, 1 \mathrm{H}, \mathrm{H}-7), 7.84(\mathrm{~s}, 1 \mathrm{H},=\mathrm{CH}-), 8.11(\mathrm{~d}, \mathrm{~J}=2.52 \mathrm{~Hz}, 1 \mathrm{H}, \mathrm{H}-5)$. 
6-bromo-3-(3'-methoxybenzylidene)chroman-4-one (3i):

IR (KBr): $1669 \mathrm{~cm}^{-1}(\mathrm{C}=\mathrm{O})$.

${ }^{1} \mathrm{H}-\mathrm{NMR}\left(\mathrm{CDCl}_{3}\right): \delta 3.84\left(\mathrm{~s}, 3 \mathrm{H}, \mathrm{OCH}_{3}\right), 5.35\left(\mathrm{~d}, \mathrm{~J}=1.84 \mathrm{~Hz}, 2 \mathrm{H},-\mathrm{OCH}_{2}-\right), 6.82-6.98(\mathrm{~m}$, $\left.4 \mathrm{H}, \mathrm{H}-8, \mathrm{H}-2^{\prime}, \mathrm{H}-4^{\prime}, \mathrm{H}-5^{\prime}\right), 7.37$ (t, 1H, H-6'), 7.57 (dd, J = $\left.2.52 \& 8.76 \mathrm{~Hz}, 1 \mathrm{H}, \mathrm{H}-7\right), 7.85$ $(\mathrm{s}, 1 \mathrm{H},=\mathrm{CH}-), 8.13(\mathrm{~d}, \mathrm{~J}=2.51 \mathrm{~Hz}, 1 \mathrm{H}, \mathrm{H}-5)$.

6-bromo-3-(4'-methylbenzylidene) chroman-4-one (3j):

IR (KBr): $1670 \mathrm{~cm}^{-1}(\mathrm{C}=\mathrm{O})$.

${ }^{1} \mathrm{H}-\mathrm{NMR}\left(\mathrm{CDCl}_{3}\right): \delta 2.41\left(\mathrm{~s}, 3 \mathrm{H}, \mathrm{CH}_{3}\right), 5.36\left(\mathrm{~d}, \mathrm{~J}=1.88 \mathrm{~Hz}, 2 \mathrm{H},-\mathrm{OCH}_{2}-\right), 6.87(\mathrm{~d}, \mathrm{~J}=8.76$ Hz, 1H, H-8), 7.21 (d, J = 8.16 Hz, 2H, H-3', H-5'), 7.25-7.27 (m, 2H, H-2', H-6'), 7.55 (dd, $\mathrm{J}=2.52 \& 8.8 \mathrm{~Hz}, 1 \mathrm{H}, \mathrm{H}-7), 7.86(\mathrm{~s}, 1 \mathrm{H},=\mathrm{CH}-), 8.12(\mathrm{~d}, \mathrm{~J}=2.56 \mathrm{~Hz}, 1 \mathrm{H}, \mathrm{H}-5)$.

6-bromo-(2',4'-dimethoxy benzylidene) chroman-4-one (3k):

IR (KBr): $1668 \mathrm{~cm}^{-1}(\mathrm{C}=\mathrm{O})$.

${ }^{1} \mathrm{H}-\mathrm{NMR}\left(\mathrm{CDCl}_{3}\right): \delta 3.85\left(\mathrm{~s}, 6 \mathrm{H}, 2 \times \mathrm{OCH}_{3}\right), 5.25\left(\mathrm{~d}, \mathrm{~J}=1.72 \mathrm{~Hz}, 2 \mathrm{H},-\mathrm{OCH}_{2}-\right), 6.49-6.54$ (m, 2H, H-3', H-5'), 6.85 (d, J = 8.8 Hz, 1H, H-8), 6.98 (d, J = 8.4 Hz, 1H, H-6'), 7.53 (dd, J $=2.56 \& 8.76 \mathrm{~Hz}, 1 \mathrm{H}, \mathrm{H}-7), 8.01(\mathrm{~s}, 1 \mathrm{H},=\mathrm{CH}-), 8.12(\mathrm{~d}, \mathrm{~J}=2.52 \mathrm{~Hz}, 1 \mathrm{H}, \mathrm{H}-5)$.

\section{Results and Discussions}

In an attempt to develop solvent free, green synthesis of 3-arylidenechroman-4-ones, a mixture of chroman-4-one, benzaldehyde and anhydrous barium hydroxide in a mortar was ground well with pestle for 5 minutes. Colour of the reaction mixture turned to yellow, indicating about the progress of reaction and was confirmed by TLC (Figure 1), when a new product was found to have formed. For the completion of the reaction, reaction mixture was left at room temperature and chroman-4-one was found to have reacted completely after 5 minutes. Reaction mixture on acidification in ice cold water gave a single solid product which was identified as $E$-3-benzylidenechroman-4-one from its spectral data and melting point comparison with literature value. Following this procedure differently substituted 3arylidenechroman-4-ones were obtained by reacting variously substituted chroman-4-ones with aromatic aldehydes (Table 1).

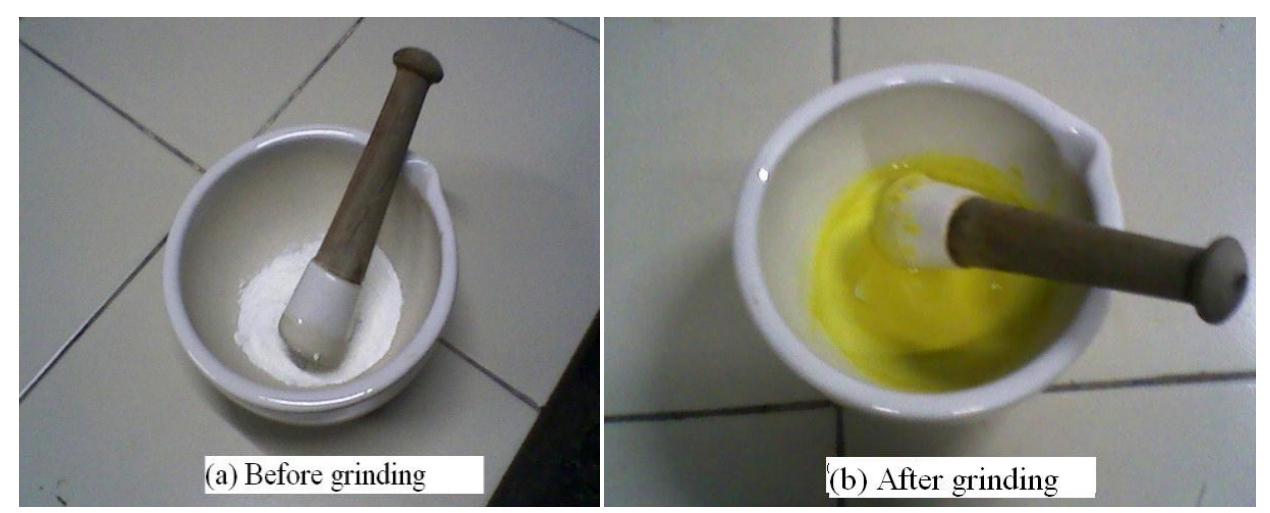

Figure 1. Photographs of the reaction mixture in mortar showing color transition taking place during reaction indicating the progress of reaction. 
The methods, reported so far make use of organic solvents either during condensation reactions or during extraction processes. Some methods though are simple but due to the prolonged refluxing are not widely accepted ${ }^{14}$. In case of piperidine, 3arylidenechroman-4-ones formed initially undergo exo-endo double bond migration to give a mixture of isomeric products ${ }^{10}$. Here in this case, the reactions take place at room temperature with enhanced rate and good selectivity. Use of toxic organic solvent was totly eliminated as no extraction was required to obtain the product.

Chroman-4-ones required for the reaction has been obtained by a modified route involving the polyphosphoric acid (PPA) catalysed cyclisation of phenoxypropanoic acid using microwave irradiations.

Table 1. Physical data of the compounds synthesized.

\begin{tabular}{cccccccc}
\hline Compounds & $\mathrm{R}$ & $\mathrm{R}_{1}$ & $\mathrm{R}_{2}$ & $\mathrm{R}_{3}$ & $\begin{array}{c}\text { Time } \\
{[\mathrm{min}]} \\
(\mathrm{x}+\mathrm{y})^{\mathrm{a}}\end{array}$ & $\begin{array}{c}\text { m.p. }{ }^{0} \mathrm{C} \\
{[\text { Lit. }]}\end{array}$ & $\begin{array}{c}\% \\
\text { yield }\end{array}$ \\
\hline $2 \mathrm{a}$ & $\mathrm{H}$ & - & - & - & - & $38-40(38.5)^{20}$ & 78 \\
$2 \mathrm{~b}$ & $\mathrm{CH}_{3}$ & - & - & - & - & $35-36(35-36)^{21}$ & 75 \\
$2 \mathrm{c}$ & $\mathrm{Br}$ & - & - & - & - & $78-79(77-79)^{22}$ & 77 \\
$3 \mathrm{a}$ & $\mathrm{H}$ & $\mathrm{H}$ & $\mathrm{H}$ & $\mathrm{H}$ & $5+5$ & $111-112(112)^{20}$ & 89 \\
$3 \mathrm{~b}$ & $\mathrm{H}$ & $\mathrm{H}$ & $\mathrm{H}$ & $\mathrm{OCH}_{3}$ & $5+5$ & $130-132(133-134)^{23}$ & 87 \\
$3 \mathrm{c}$ & $\mathrm{H}$ & $\mathrm{H}$ & $\mathrm{OCH}$ & $\mathrm{H}$ & $5+10$ & $103-104(103-104)^{24}$ & 85 \\
$3 \mathrm{~d}$ & $\mathrm{CH}$ & $\mathrm{H}$ & $\mathrm{H}$ & $\mathrm{H}_{3}$ & $5+10$ & $146-147(147-148)^{25}$ & 87 \\
$3 \mathrm{e}$ & $\mathrm{CH}$ & $\mathrm{H}$ & $\mathrm{H}$ & $\mathrm{CH}_{3}$ & $5+10$ & $126-28$ & 83 \\
$3 \mathrm{f}$ & $\mathrm{CH}$ & $\mathrm{H}$ & $\mathrm{H}$ & $\mathrm{OCH}_{3}$ & $5+10$ & $108-10$ & 88 \\
$3 \mathrm{~g}$ & $\mathrm{Br}$ & $\mathrm{H}$ & $\mathrm{H}$ & $\mathrm{H}$ & $5+10$ & $158-60$ & 92 \\
$3 \mathrm{~h}$ & $\mathrm{Br}$ & $\mathrm{H}$ & $\mathrm{H}$ & $\mathrm{OCH}_{3}$ & $5+10$ & $166-67$ & 91 \\
$3 \mathrm{i}$ & $\mathrm{Br}$ & $\mathrm{H}$ & $\mathrm{OCH}$ & $\mathrm{H}_{3}$ & $5+10$ & $112-13$ & 88 \\
$3 \mathrm{j}$ & $\mathrm{Br}$ & $\mathrm{H}$ & $\mathrm{H}$ & $\mathrm{CH}_{3}$ & $5+10$ & $156-58$ & 86 \\
$3 \mathrm{k}$ & $\mathrm{Br}$ & $\mathrm{OCH}$ & $\mathrm{H}$ & $\mathrm{OCH}_{3}$ & $5+15$ & $168-69$ & 89 \\
\hline $\mathrm{x}$ & & & & & & \\
\hline
\end{tabular}

${ }^{\mathrm{a}} \mathrm{x}$ - Grinding time, $\mathrm{y}$ - time for which reaction mixture was kept at room temperature.

\section{Conclusion}

This appears to be a simple and highly efficient procedure for the synthesis of 3arylidenechroman-4-ones as it requires much shorter time for the completion of the reaction and yields of the products are high. More over it is simple and eco-friendly protocol as it avoids the use of solvent at any stage of reaction or isolation process.

\section{Acknowledgements}

The authors are grateful to CSIR (New Delhi) for providing financial support. 


\section{References}

1. (a) Ellis G P, Chromenes, Chromanones, and Chromones, John Wiley \& Sons, New York, 1977, 214-452; (b) Jiang H B, Huang J, Guo M J, Zou P and Tian X Q, Acta Pharmaceutica Sinica, 2007, 42, 118.

2. (a) Flavin, M T, Rizzo J D, Khilevich A, Kucherenko A, Sheinkman A K, Vilaychack V, Lin L, Chen W, Greenwood E M, Pengsuparp T, Pezzuto J M, Hughes S H, Flavin T M, Cibulski M, Boulanger W A, Shone R L and Xu Z Q, J. Med. Chem., 1996, 39, 1303; (b) Xu Z Q, Bucheit R W, Stup T L, Flavin M T, Khilevich A, Rezzo J D, Lin L and Zembower D E, Bioorg. Med. Chem. Lett., 1998, 8, 2179; (c) Ishikawa T, Oku Y, Tanaka T and Kamamoto T, Tetrahedron Lett., 1999, 40, 3777.

3. Ravise A and Kirkiacharian B S, Phytopathol., 1978, 92, 36.

4. Nakib T A, Bezjak V, Meegan M J and Chandy R, Eur. J. Med. Chem., 1990, 25, 455.

5. Darmanaden R, Dhanutrito H, Castel J, Loubatiere J and Flandare O, II Farmaco, 1984, 39, 876.

6. Kirkiacharian B S, Gomis M and Koutsourakis P G, Eur. J. Med. Chem., 1989, 24, 309.

7. Foroumadi A, Kermani A S, Emami S, Dehghan G, Sorkhi M, Arabsorkhi F, Heidari M R, Abdollahi M and Shafiee A, Bioorg. Med. Chem. Lett., 2007, 17, 6764.

8. $\quad$ Srikanth N, Kon O L, Ng S C and Sim K Y, J. Chem. Res.(S), 1997, 202.

9. Mulvagh D, Meegan M J and Donnelly D M X, J. Chem. Res. (S), 1979, 137.

10. Levai, A, Arkivoc, 2004 (vii) 15.

11. Sangwan N K and Rastogi S N, Indian J. Chem., 1981, 20B, 135.

12. Wagner G, Garbe C, Vieweg H, Brunn M and Dittrich A, Pharmazie, 1979, 34, 55.

13. Ellis G P, Chromenes, Chromanones, and Chromones, John wiley \& Sons, New York 1977, 298.

14. (a) Levai A and Szabo Z, Pharmazie, 1992, 47, 56; (b) Farkas L, Gottsegen A, and Nogradi M, Tetrahedron, 1970, 26, 2787.

15. Mondal T K, Pal R, Mondal R and Mallika A K, E. J. chem., 2011, 8 (2) 863.

16. Basavaiah D, Bakthadoss M and Pandiaraju S, Chem. Commun., 1998, 1639.

17. (a) Varma R S, Green Chem. Lett. Rev., 2007, 1, 37; (b) Cave G W V, Raston C L and Scott J L, Chem. Commun., 2001, 2159; (c) Nalimi H, Sharghi, H, Salimi F and Rabiei K, Heteroatom Chem., 2008, 19, 43.

18. (a) Kumar D, Sekhar K V G C, Dhillon M, Rao V Sand, Varma R S, Green Chem. 2004, 6 , 156; (b) Sheikhan N, Mirjalili B F, Hajipour A and Bamoniri A, Acta. Chim. Slov., 2008, 55, 209; (c) Reza Kiasat A and Fallah-Mehrjardi M, J. Braz. Chem. Soc., 2008, 19, 1595; (d) Li J T and Sun S F, E. J. chem., 2010,7 (3), 922.

19. (a) Kumar S, Lamba M S and Makrandi J K, Green Chem. Lett. \& Rev., 2008, 1, 123; (b) Jakhar K and Makrandi J K, Green Chem. Lett. \& Rev., 2008, 1, 219, Sharma D, Kumar S and Makrandi J K, Green Chem. Lett. \& Rev., 2011, 4, 127.

20. Powell S G, J. Am. Chem. Soc., 1923, 45, 2708.

21. Powell S G and Johnson N G, J. Am. Chem. Soc., 1924, 46, 2861.

22. Wiley P F, J. Am. Chem. Soc., 1951, 73, 4205.

23. Chatterjea J N, Shaw S C and Singh J N, J. Indian Chem. Soc., 1974, 51, 281.

24. Pfeiffer P, Grimm K and Schmidt H, Justus liebigs Ann. Chem., 1949, 564, 208.

25. Liebermann C and Lindenbaum S, Ber., 1909, 42, 1392. 


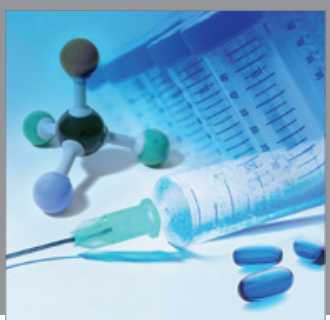

International Journal of

Medicinal Chemistry

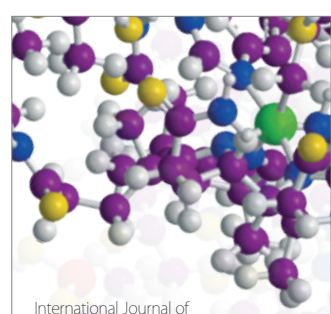

Carbohydrate Chemistry

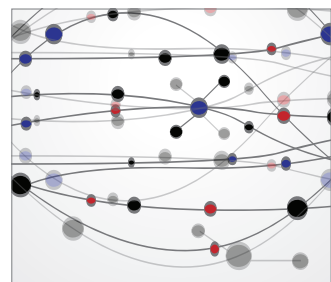

The Scientific World Journal
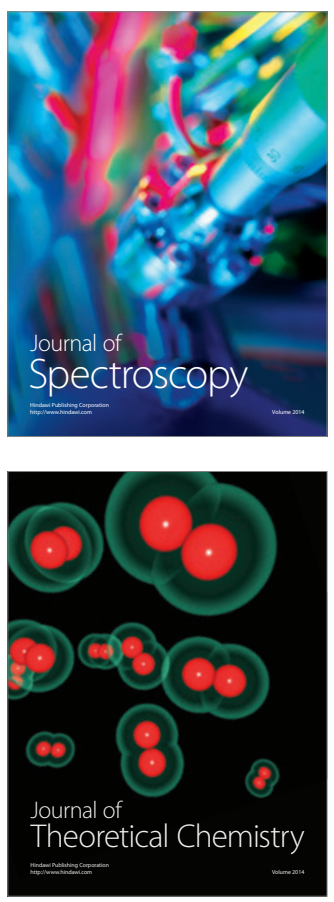
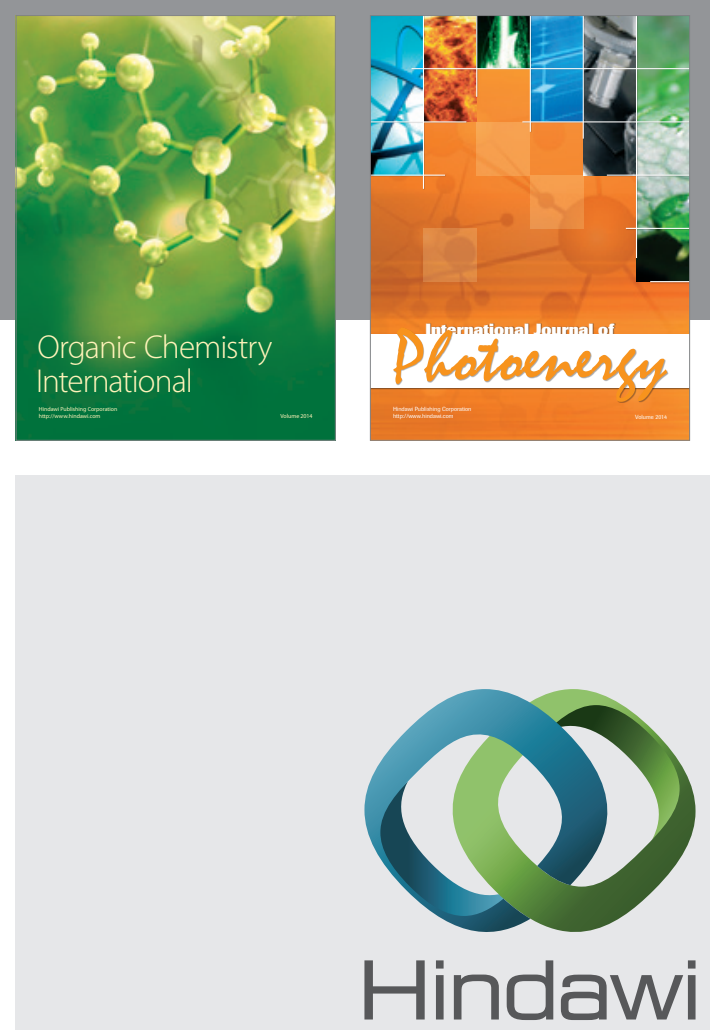

Submit your manuscripts at

http://www.hindawi.com
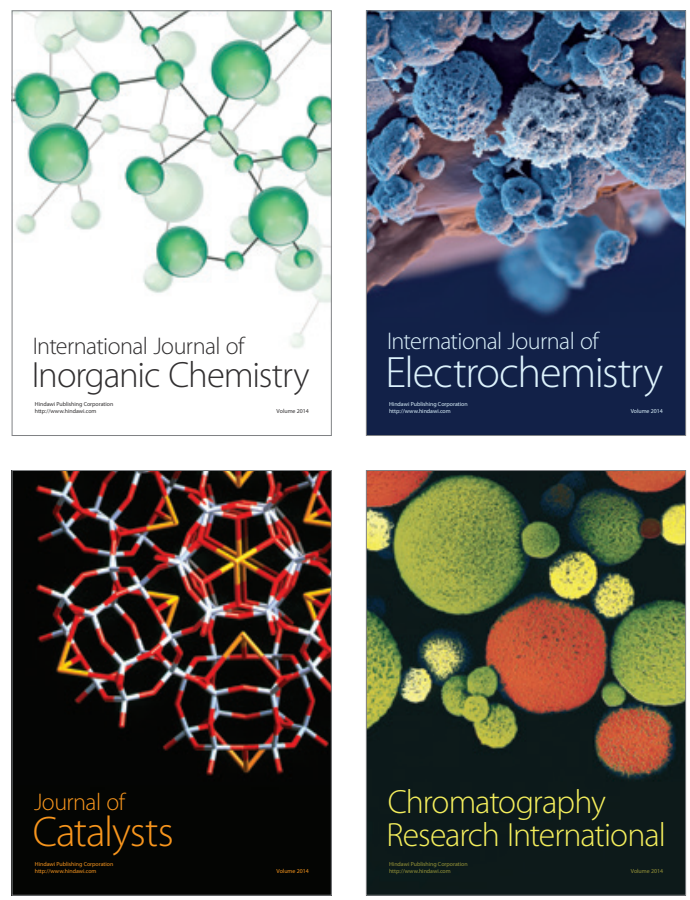
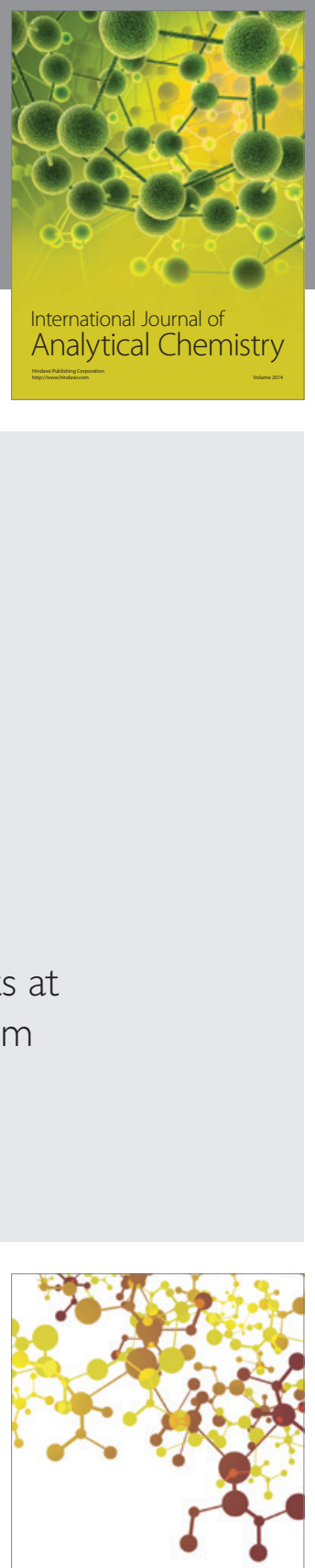

Journal of

Applied Chemistry
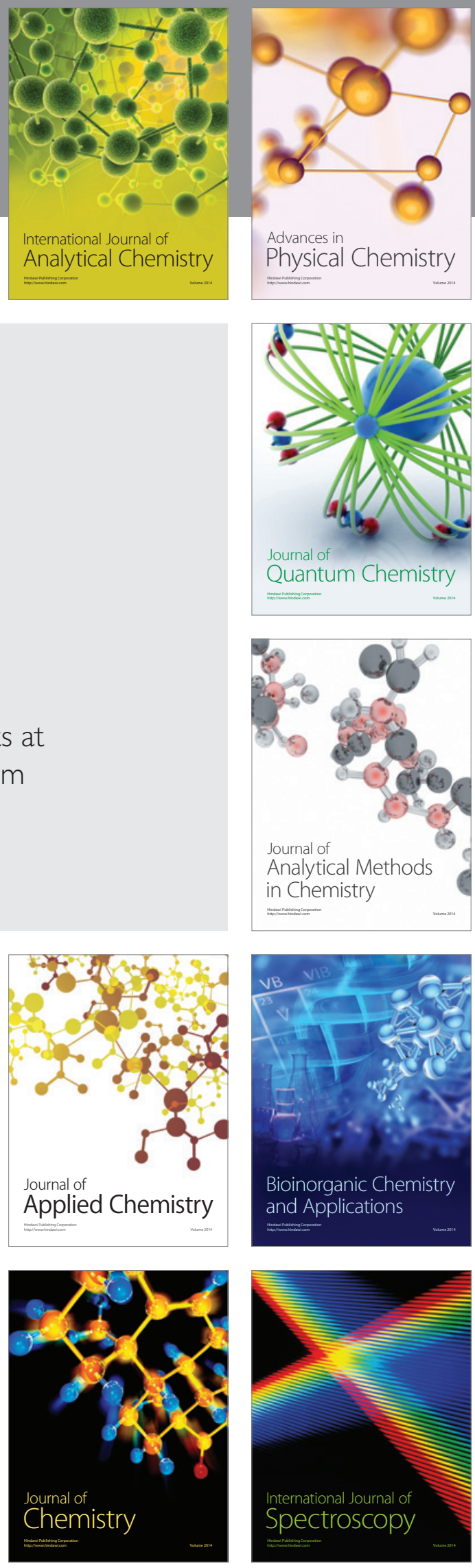